\title{
BMJ Open Importance and role of independent data monitoring committees (IDMCs) in oncology clinical trials
}

\author{
P Schöffski
}

To cite: Schöffski P. Importance and role of independent data monitoring committees (IDMCs) in oncology clinical trials. BMJ Open 2021;11:e047294. doi:10.1136/ bmjopen-2020-047294

- Prepublication history for this paper is available online. To view these files, please visit the journal online (http://dx.doi. org/10.1136/bmjopen-2020047294).

Received 24 November 2020 Accepted 24 September 2021

Check for updates

(c) Author(s) (or their employer(s)) 2021. Re-use permitted under CC BY-NC. No commercial re-use. See rights and permissions. Published by BMJ.

Department of General Medical Oncology, University Hospitals Leuven, KU Leuven, Leuven Cancer Institute, Leuven, Belgium

Correspondence to Dr P Schöffski; patrick.schoffski@uzleuven.be

\section{ABSTRACT}

The role and use of independent data monitoring committees (IDMCs) has evolved over the past decades. The Food and Drug Administration and European Medicines Agency have issued guidelines on the role and functioning of such committees. In general, data monitoring committees are recommended for large, often randomised clinical trials involving life-threatening diseases, studies performed in vulnerable populations or where the experimental intervention can potentially harm the trial participant. Such committees play an important role in trials evaluating treatments with the potential to prolong life or reduce the risk of major adverse health outcomes.

Typically, oncology clinical trials fall within these recommendations, as they are often large, randomised, multicentric protocols aiming at improving survival outcomes by exploring the use of study treatments that may be associated with a significant risk of serious, even life-threatening adverse events. IDMCs are required for National Cancer Institute phase III randomised trials, European Organisation for Research and Treatment of Cancer phase II/III trials with formal interim analyses, early-stopping rules or adaptive studies. The primary role of an IDMC of ensuring the safety of study participants and maintaining clinical trial integrity is particularly important in oncology trials, due to the nature of the disease, the potential for treatment toxicity and for instilling confidence that the clinical trial data are reliable. A clear understanding by IDMC members of the natural course of the disease, treatment landscape, importance and relevance of certain adverse events in trial participants, clinical trial methodology in general and stopping rules for oncology trials in particular, is crucial for the functioning of an IDMC.

It is recommended that IDMC members should be experienced trialists, have a track record of strong clinical, statistical and/or methodological expertise and the required level of independence, as they play a highly important role in the protection of study participants, and in commercially and strategically important go/no decisions. Ideally, IDMC members should have relevant experience or have some training, mentorship or guidelines.

\section{INTRODUCTION}

Data monitoring committees (DMCs) can be traced back to 1967 and the 'Greenberg

\section{Strengths and limitations of this study}

The strengths of independent data monitoring committees (IDMCs) are in ensuring the safety of clinical trial participants, maintaining trial integrity and instilling confidence in the reliability of trial data.

- Furthermore, IDMCs can be extremely helpful when using early stopping assessments to ensure that as trial data is accumulated, the risk/benefit balance continues to be in favour of the trial participants.

- In oncology clinical trials, especially those involving rare cancers or those with complex or adaptive designs, IDMCs can provide valuable input on decisions pertaining to study progress, proper study conduct and the interpretation of findings.

- With the increasing use of IDMCs, a limitation is the potential lack of enough trained or experienced committee members to adequately fufill the roles required.

- Another limitation is the difficulty of establishing a fully independent IDMC that simulatenously has members who are highly knowledgeable and experienced experts.

Report', and in 1979 the National Institutes of Health (NIH) in the USA issued a policy that 'every clinical trial should have prevision for data and safety monitoring'. ${ }^{1-3}$ In the 1990s, the US Food and Drug Administration (FDA) reviewed operational aspects of safety monitoring in clinical trials, the NIH further developed a policy on data and safety monitoring committees, and guidance documents by the International Conference on Harmonisation (ICH) also included references to DMCs. ${ }^{14}$

DMCs were initially used in large randomised multicentre clinical trials to allow for interim safety monitoring of study data to ensure the ongoing safety of trial participants. ${ }^{3}$ More recently, DMCs have been increasingly used in industry sponsored trials, from phase I to III. ${ }^{3}$

The use and role of DMCs has evolved over the past decades. In 1990, only $10 \%$ of trials published in medical journals reported using DMCs compared with $25 \%$ in $2000 .^{5}$ A report 
of an analysis of the ClinicalTrials.gov database in 2010 of trials involving indications such as cardiovascular disease, oncology and mental health, showed that $41 \%$ of registered trials reported using a DMC. ${ }^{5}$

DMCs are also known as data and safety monitoring boards/committees (DSMCs), treatment effects committees or independent DMCs (IDMCs) for highlighting the independent functioning of members of the committee. ${ }^{36-8}$ Other names include monitoring committee (MC), data and ethics monitoring committee and safety MC. ${ }^{8}$ This very heterogeneous and sometimes confusing nomenclature is a reflection of the variable responsibilities of such committee, which are not defined by name, but by a charter for each specific trial. For the purposes of this manuscript, reference will be made to IDMCs.

The IDMC usually consists of a group of independent experts that evaluate the accumulating data from a clinical trial with regard to the progress, safety of study participants, critical efficacy endpoints of the trial and make recommendations regarding trial modification, continuation or termination. ${ }^{39}$ The function of the IDMC is not to be confused with that of a typical study steering committee (SSC) (also known as the steering committee, clinical trial steering committee, study executive committee or study executive board). ${ }^{3910}$ The SSC may be responsible for designing the trial, has the executive power over the trial, provides overall supervision and maintains the quality of trial conduct. ${ }^{3910}$ While the IDMC may receive unblinded data in order to make their recommendations and is typically composed of experts not involved in the trial itself, the SSC members play a very active role, for example, as investigators, but remain blinded while the study is ongoing. ${ }^{910}$

In the last two decades, various health authorities have issued guidance documents on the use of IDMCs in clinical trials. The FDA issued a finalised guidance document in 2006 on 'Guidance for Clinical Trial Sponsors, Establishment and Operation of Clinical Trial DMCs. ${ }^{13}$ The European Medicines Agency (EMA) issued a 'Guideline on DMC' that was adopted by the Committee for Medicinal Products for Human Use (CHMP) in July 2005 and the guidelines came into effect in January $2006 .{ }^{9}$ These official documents highlight the importance of independent data monitoring and the regulatory relevance of the composition and functioning of IDMCs.

\section{THE NEED FOR AN IDMC, REGULATORY REQUIREMENTS AND THE TYPES OF CLINICAL TRIALS THAT BENEFIT THE MOST FROM IDMCs}

Safety monitoring is required for all clinical trials throughout the duration of the study, however, not all clinical trials require an IDMC. ${ }^{3}$ Currently, the FDA regulations do not impose specific requirements for the use of IDMCs. ${ }^{36}$ The FDA provides nonbinding guidance recommendations on the need for an IDMC, and the recommendations by the FDA and EMA for when an IDMC should be used in a trial are shown in table $1 .{ }^{369}$ Generally, there is concordance between the FDA and EMA guidelines that the use of an IDMC in a clinical trial is recommended for any controlled clinical trial examining mortality or major morbidity, or for large, randomised, trials with vulnerable populations, or multicentre clinical trials evaluating treatments to prolong life or reduce risk of major adverse health outcomes (eg, cardiovascular events or recurrence of cancer). ${ }^{369}$

Various institutes, organisations and academic bodies have their own requirements for when a trial should include an IDMC. ${ }^{41011}$ For example, the NIH require data and safety monitoring for phase III clinical trials; and recommend IDMCs for certain phase I and II studies - such as in trials that are multicentred, blinded, or include vulnerable populations or high-risk interventions, etc. ${ }^{12}$ The ICH Guideline for Good Clinical Practice (GCP) E6 (R1) mentions that sponsors can establish an IDMC to evaluate at intervals the clinical trial progress, safety data, critical efficacy endpoints and make recommendations to the trial sponsor to continue, stop or modify a trial. ${ }^{13}{ }^{14}$ Such sponsor can be a commercial or an academic sponsor. In the WHO guideline on IDMCs, it states that IDMCs are critical for clinical trials that save lives, reduce the risk of a major adverse health outcome, prevent serious disease progression and are important where interim analyses are needed for protecting the safety of study participants. ${ }^{8}$ Furthermore, the WHO also states that IDMCs are considered relevant for controlled clinical trials with mortality/morbidity endpoints of those that reduce severe morbidity or mortality, early trials with high-risk interventions, early trials with a novel intervention and limited efficacy and safety information, trials with complex data accrual or where data accrual may impact on study design and participant safety - particularly in trials of long-duration, trials with early-stopping rules, emergency situation trials and trials involving vulnerable populations. ${ }^{8}$ All of this often applies to typical clinical studies in oncology.

\section{Oncology clinical trials and the need for an IDMC}

Typical oncology clinical trials involve life-threatening diseases, are often multicentred, of long duration with outcomes that may include progression-free survival or overall survival (OS) endpoints, with study treatments that may be associated with serious, sporadically fatal adverse events and commonly include preplanned interim analyses. $^{3913}$

The US National Cancer Institute (NCI) has a continuum scale for the types of monitoring required for clinical trials that are either performed or supported by the NCI. ${ }^{15}$ While all NCI trials require some form of monitoring, trials that have higher risks or are of greater size and complexity, such as many oncology trials, require monitoring by an IDMC (figure 1)..$^{15}$

Table 2 shows when IDMCs are needed or recommended in oncology trials that are supported or 
Table 1 Recommendations by North American and European regulatory authorities for determining the need for an IDMC in clinical trials ${ }^{39}$

Considerations
Emergency settings with no informed consent
Risk to clinical trial participant, that is,
High safety concerns.
Highly favourable or unfavourable study
endpoint.
Particular safety concerns (eg, particularly
invasive procedure).
- Possible serious toxicity with study treatment.
Potentially fragile or vulnerable populations.
- Study participants with elevated risk of death or
other serious outcomes.
A large, multicentre clinical trial of long duration.

Practicality of IDMC, that is,

Clinical trial of short duration.

May be impractical

Short-term trials with high risks (ie, important safety concerns).

Ensuring scientific validity and perception of the trial, that is,

- Trials with appreciable duration. $\dagger$

- Compelling new external information that may impact on the trial.

- Accumulating trial data that may indicate the need for modifications to be made.

Clinical trial examining lesser outcomes (eg, symptom relief)‡

\section{Consider}

Consider

\begin{tabular}{|c|c|c|}
\hline FDA $^{3}$ & Considerations & EMEA/EMA ${ }^{9}$ \\
\hline \multicolumn{3}{|l|}{ Required } \\
\hline Recommended & $\begin{array}{l}\text { Risk to clinical trial participant, that is, } \\
\text { Life-threatening diseases } \\
\text { (regardless of whether the study } \\
\text { treatment reduces morbidity or } \\
\text { mortality, or relieves the patient's } \\
\text { situation). } \\
\text { Types of patient population (eg, } \\
\text { paediatric or mentally disabled } \\
\text { patients). } \\
\text { Possible ability of study treatment } \\
\text { to harm the patient. }\end{array}$ & Recommended \\
\hline
\end{tabular}

Practicality of IDMC, that is,

- Clinical trial of short duration and the study drugs are well characterised and known to not harm the patient.

Ensuring scientific validity and

perception of the trial, that is,

May be

- Long-term trials (even if non-life threatening disease).

- Study design with preplanned interim analyses for early stopping.

- Accumulating trial data that may indicate the need for modifications to be made.

*For example, study participants who may be children, or pregnant women, or very elderly, or terminally ill, or have diminished mental capacity.

†Trials that are conducted over a long period of time may be affected by changes over time (ie, changes in the standard of treatment, or in the understanding of the disease, etc). These external changes may prompt an interest in modifying some aspects of the trial as it progresses. $\ddagger$ Unless there is an elevated risks of more severe outcomes.

EMA, European Medicines Agency; EMEA, European Medicines Evaluation Agency; FDA, Food and Drug Administration; IDMC, independent data monitoring committee.

performed by either the NCI or the European Organisation for Research and Treatment of Cancer (EORTC). ${ }^{1015}$

There is concordance between the FDA, EMA, NCI and EORTC in their recommendations of an IDMC for large, randomised clinical trials. ${ }^{391015}$ The FDA, EMA and EORTC guidelines recommend the use of IDMCs for study treatments that have safety concerns or may have the ability to harm the patient. ${ }^{3910}$ In EORTC clinical trials, IDMCs are mandatory for clinical trials with formal interim analyses or early stopping rules, or planned adaptations to the sample size or the study design, while in the FDA and EMA guidelines an IDMC should be considered or may be indicated, respectively. ${ }^{3} 10$

\section{THE EVOLVING ROLE AND IMPORTANCE OF AN IDMC IN ONCOLOGY CLINICAL TRIALS}

Over the past decades, the use of IDMCs has increased and evolved, which may include early termination decisions, evaluations of interim data or adaptive trials with predefined sample size or trial design modification. $^{15} 101316-18$

In oncology clinical trials, IDMCs are important due to the severity of the disease under study and the incidence and potential severity of adverse events that may be caused by cancer treatments. ${ }^{13}$ Mortality rates are often high in oncology clinical trials and many of these studies require very long-term follow-up. ${ }^{13}$ Oncology clinical trials increasingly involve rare cancers which can have an 


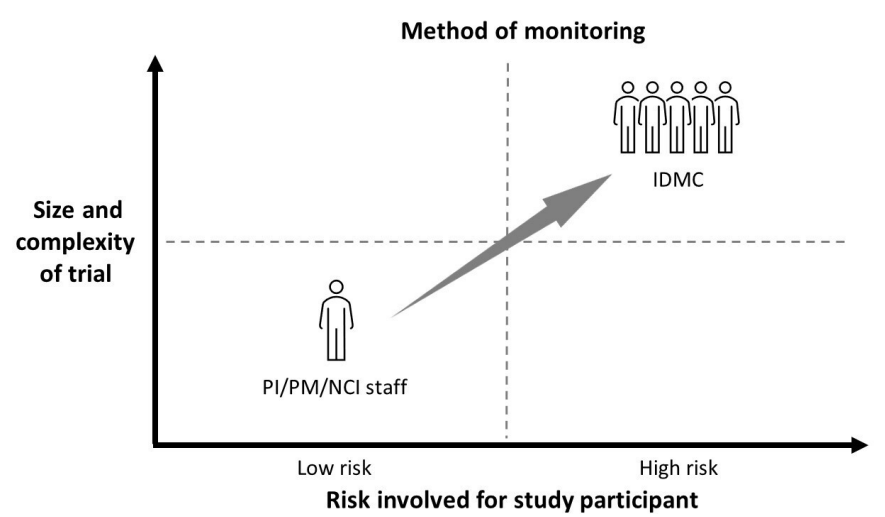

Figure 1 Types of monitoring required for clinical trials performed or supported by the $\mathrm{NCl}$. Adapted from information in: policy of the National Cancer Institute for Data and Safety Monitoring of Clinical Trials. ${ }^{15}$ IDMC, independent data monitoring committee; $\mathrm{NCl}$ staff, National Cancer Institute programme staff; PI, principal investigator; PM, project manager.

effect on patient accrual and the use of IDMCs in such trials may provide valuable input on decisions pertaining to study progress, particularly if enrollment is slow and new treatments become available during the trial period that could benefit the patient. Many modern oncology trials explore novel treatments in 'niche' indications, where studies have to be designed in the absence of reliable references from earlier trials. Trials in new oncology settings are often applying innovative endpoints such as patient-reported outcomes, where rigorous guidance may be required to ensure proper study conduct and interpretation of findings.

The primary role of an IDMC is to ensure the safety of study participants and maintain clinical trial integrity. ${ }^{1319}$ The IDMC plays a vital role in instilling confidence that the clinical trial data are reliable. ${ }^{20}$ Table 3 summarises the characteristic responsibilities of an IDMC. 35681016 19-22

To support the IDMC's primary role of protecting study participants, IDMCs are usually involved in early stopping assessments (for toxicity, futility or efficacy) to ensure that as data are accumulated, the risk/benefit balance continues to be in favour of potentially benefitting the trial participants. ${ }^{13} 1921$ In oncology clinical trials the ongoing risk/benefit assessment is pertinent. After reviewing the accumulated data, the IDMC makes recommendations on whether the clinical trial should be continued or terminated and because this is a major decision, the predefined rules about early stopping provide important guidance to the IDMC. ${ }^{13} 1921$

IDMC members are in the unique and privileged position of having access to interim unblinded data of a clinical trial and they have an obligation to act on that information to safeguard study participants, while at the same time maintain the integrity of the study with all partners involved (trial participants, investigators, sponsor, authorities). ${ }^{23-25}$ IDMC members draw conclusions on a complex set of skills, knowledge and experience, and the issues they will face have been described as 'the toughest job in clinical medicine'. ${ }^{23} 16$ In order for IDMC members to adequately fulfil their role, experienced member are required or training is needed to prepare the next generation of IDMC members. ${ }^{16} 23$ Members participating in IDMCs require: expertise in the disease area and treatment landscape, clinical trial and GCP knowledge, and IDMC training (eg, IDMC responsibilities, understanding of statistical stopping rules). ${ }^{23}$

\begin{tabular}{|c|c|c|c|}
\hline $\begin{array}{l}\text { Clinical trials supported or } \\
\text { performed by the } \mathrm{NCI}^{15}\end{array}$ & $\begin{array}{l}\text { Responsibility for data } \\
\text { and safety monitoring }\end{array}$ & EORTC clinical trials ${ }^{10}$ & $\begin{array}{l}\text { Responsibility for data } \\
\text { and safety monitoring }\end{array}$ \\
\hline $\begin{array}{l}\text { All phase III randomised } \\
\text { clinical trials }\end{array}$ & IDMC mandatory & $\begin{array}{l}\text { Trials designed with planned adaptions of the sample } \\
\text { size or of the study designt }\end{array}$ & IDMC mandatory \\
\hline \multirow{2}{*}{ Co-funded with other NIH IC } & & $\begin{array}{l}\text { Pivotal clinical trials that will be used for drug } \\
\text { registration purposes }\end{array}$ & IDMC recommended \\
\hline & & $\begin{array}{l}\text { Clinical trials requiring: } \\
>>1000 \text { patients, or } \\
>4 \text { years of patients accrual. }\end{array}$ & IDMC recommended \\
\hline
\end{tabular}

*Dependent on continuum for monitoring by the $\mathrm{NCl}$ (see figure 1).

†That are based on interim analysis of the unblinded results of the trial.

EORTC, European Organisation for Research and Treatment of Cancer; NIH IC, National Institutes of Health Institutes or Centres; IDMC, independent data monitoring committee; $\mathrm{NCl}$, National Cancer Institute; PI, principal investigator; PM, project manager; NCl staff, National Cancer Institute programme staff. 
Table 3 Responsibilities of an IDMC $3568101619-22$

\begin{tabular}{|c|c|}
\hline Responsibility & Required \\
\hline Protocol review & Regularly \\
\hline Protocol approval & Possibly* \\
\hline Familiarisation with clinical trial protocol and data collection plan & Always \\
\hline Maintain independence from sponsor & Always \\
\hline Patient accrual assessment and recommend modifications if needed & Always/almost always \\
\hline $\begin{array}{l}\text { Data quality assessment (eg, incomplete data, drop-out rate, trial operational conduct) and } \\
\text { recommend modifications if needed }\end{array}$ & Always/almost always \\
\hline $\begin{array}{l}\text { Safety assessments (including review of: adverse events overall and by treatment arm, cumulated } \\
\text { serious and unexpected adverse events, etc) }\end{array}$ & Always \\
\hline $\begin{array}{l}\text { Safety recommendations (eg, protocol amendments, increased surveillance, changing product } \\
\text { dosaging, additional training or procedures, early stopping) }\end{array}$ & Always \\
\hline Review efficacy outcomes & Always/almost always \\
\hline Assess/consider the relevence and impact of external evidence & Always/almost always \\
\hline $\begin{array}{l}\text { Early trial termination recommendation or risk/benefit assessments (eg, stopping for harm, or stopping } \\
\text { for benefit, or stopping for futility) }\end{array}$ & Always/almost always \\
\hline Maintain confidentiality of interim results & Always \\
\hline $\begin{array}{l}\text { Maintain confidential meeting minutes from each IDMC meeting, which remain uncirculated until the } \\
\text { trial is terminated/completed }\end{array}$ & Always/almost always \\
\hline $\begin{array}{l}\text { Provision of a written recommendation from each IDMC meeting regarding whether a protocol should } \\
\text { be amended and if a study should continue or be terminated }\end{array}$ & Always \\
\hline Study presentation review & Possibly* \\
\hline Study presentation approval & Possibly* \\
\hline Study manuscript/publication review & Possibly* \\
\hline Study manuscript/publication approval & Possibly* \\
\hline
\end{tabular}

Adapted from DeMets and Ellenberg. ${ }^{19}$

*Depends on IDMC charter or institute's/organisation's IDMC policy.

IDMC, independent data monitoring committee.

\section{IMPLEMENTATION OF IDMCs IN ONCOLOGY CLINICAL TRIALS Establishing an IDMC and composition}

In oncology clinical trials, the trial steering committee, sponsor, principal investigator or project manager will usually appoint IDMC members, for a defined term. ${ }^{13} 1526$ Many sponsors have a standard operating procedure for identifying possible IDMC members. ${ }^{16}$ For NCI studies, proposed IDMC members have to be approved by the awarding NCI division director or designee. ${ }^{15}$ The voting members represent the interest of the patients. ${ }^{15}$ For EORTC studies, the EORTC Board nominate the permanent IDMC chair and members for a term of 3 years; EORTC's IDMC chair carries responsibilities for a range of ongoing studies, while commercial sponsors commonly appoint one IDMC/one chair per protocol. ${ }^{10}$ In EORTC studies, the IDMC chair decides on study-specific external IDMC members - whose names may be proposed by the study coordinator, or the IDMC secretariat, or by the IDMC chair themself. ${ }^{10}$ In company-sponsored trial, the sponsor usually applies their own rules for selecting, appointing and contracting IDMC chairs and members, but defines the required expertise and the responsibilities of a given IDMC member in a protocol-specific charter.
The IDMC members for an oncology trial may comprise of various disciplines, such as medical oncology/surgical oncology/radiation oncology, biostatistics, other physicians or scientists, pathology specialists, medical ethicist and lay representatives may also be included, based on their experience, knowledge and relevance in relation to the clinical trial, their experience on serving on IDMCs, and in the absence of conflict of interest. ${ }^{310} 1315$

The composition of the IDMC is critical for correct decision making and to avoid overlooking problems on the trial that may need addressing. ${ }^{3}$ The number of members of the IDMC can vary depending on the clinical trial, institute, or organisation. For example, EORTC requires at least five permanent members, the Radiation Therapy Oncology Group requires at least ten IDMC voting members for phase III trials (including medical subspecialties, ethics, statistics and patient advocacy). ${ }^{1025}$ In general, the number of members in an IDMC typically ranges from 3 to 15 people (usually involving an odd number to facilitate definite decisions when voting), with more complicated studies often requiring a larger number of members. ${ }^{1326}$ However, the number of members in an IDMC should be neither too large (ie, it becomes difficult 
to identify a meeting time when everyone is available, or discussions become too unwieldy), nor too small (ie, specific expertise that may be needed could be missing). ${ }^{13}$ To some extent, the complexity of the oncology clinical trial, the type of treatment being evaluated, and distinct anticipated adverse effects may guide the choice of the type and number of subspecialist members. Outside expertise may also be called on in specific situations, usually on suggestion of IDMC members and coordinated by the sponsor. All this is typically described in the IDMC charter. ${ }^{25}$

Two key positions on an IDMC are the chair and biostatistician. ${ }^{1}$ The IDMC chair is usually elected from the voting member of the IDMC. ${ }^{10}{ }^{15}$ The chair leads the IDMC and is responsible for developing the agenda of the meetings, overseeing the meetings and communicating the findings of the IDMC meeting to the steering committee or sponsor within predefined timelines. ${ }^{1} 27$ The chair should have substantial prior IDMC experience and is often an expert in the disease under study investigation. ${ }^{1}$ The biostatistician also plays a crucial role in IDMC deliberations, and in addition to expertise in clinical research design and analysis, ideally should have experience with the disease nuances that are being addressed in the particular clinical trial. ${ }^{14}$

\section{Independence}

IDMC members should be independent and free from any conflicts of interest relating to the clinical trial outcome, trial sponsor, stock ownership, patent ownership, etc, and should not have entered patients on the trial or have been involved in the trial design. ${ }^{3} 1013152027$ For EORTC permanent and study-specific IDMCs, conflicts of interest also include being a principal investigator of a competing study, or being a permanent member on various review committees, or being on an advisory board for: (1) either the same drug under investigation or (2) for the same indication in the study and the same pharmaceutical company. ${ }^{10}$

IDMC members disclose any potential conflicts of interests when invited to serve on an IDMC, and if potential conflicts develop during the member's tenure on the IDMC, these too must be disclosed in a continuous manner. ${ }^{3} 15$ Permanent EORTC IDMC members, for example, complete and sign the EORTC confidentiality agreement and interest disclosure form on joining the IDMC and for each IDMC meeting. ${ }^{10}$

A conundrum exists between a fully independent DMC (which has no involvement in trial design or conduct, except through their charter-defined IDMC responsibilities, and no financial connection with the sponsor, other than compensation for their IDMC role, or other conflict of interest) vs having the most knowledgeable and experienced researchers as IDMC members (who most probably will have been involved or have some connection with other trials in that area). ${ }^{317}$ Highly knowledgeable and experienced experts are desirable for IDMCs, but are also more likely to have possible conflicts of interest due to their involvement in many clinical trials, advisory boards, speaker engagements, etc and these need to be managed. ${ }^{1720}$ The FDA defines independence on a continuum, and while IDMCs are rarely entirely independent, the FDA also recommends that the IDMC member should not be involved in the conduct of the trial in any way (other than in their role as an IDMC member) and that the IDMC member should generally have "no ongoing financial relationship with the trial's commercial sponsor, ${ }^{3}$ While it may not be possible to completely eliminate all conflicts of interest, there should not be significant financial, intellectual/professional advancement or other gains that are linked to the trial outcomes for the IDMC members or their family members. ${ }^{40}$ If conflicts of interest are minor, they should be managed through disclosure. ${ }^{26}$ The IDMC charter should clearly describe the independence of the DMC for the trial and what is construed as a conflict of interest. ${ }^{20}$

The advantage of independence of a DMC is the promotion of objectivity and the reduction of bias, which will increase the credibility of a trial and promote patient benefits. ${ }^{3}$ Another advantage is that the independence of the IDMC members may facilitate taking critical decisions, that may not be in primary best interest of sponsor or involved investigators.

\section{IDMC charter}

The IDMC charter is crucial for outlining the standard operating procedure of the committee, and the agreed roles and responsibilities of committee members (table 4). ${ }^{1391327-29}$ The content of the charter may vary from trial to trial, as it addresses specifics of each given research project. Similar to the protocol and analytical plans, the charter is an important document and depending on the trial - may be required by regulatory bodies prior to the initiation of a trial. ${ }^{3}$ A charter template has been proposed by the DAMOCLES Study Group for helping IDMCs to do their job. ${ }^{29}$

\section{IDMC responsibilities in oncology clinical trials and IDMC meetings}

The responsibilities of IDMCs in general have been outlined previously and are presented in table 3. At IDMC meetings, the members will review the clinical trial data and analyse cumulative toxicity, and depending on what is specified in the charter, may determine if the treatment plan and protocol is being adhered to, assess patient accrual, review interim analyses and evaluate end-point or stopping-point rules. ${ }^{315} 27$ In oncology clinical trials, in addition to safety interim data assessments and evaluating the conduct and progress of the clinical trial, IDMCs often also assess clinical benefit, especially in trials involving novel or surrogate endpoints, or patient-reported outcomes. ${ }^{121}$ Critical decisions in such trials should not be taken lightly, and a clear understanding of the stopping rules for the trial is needed. In oncology clinical trials, special consideration needs to be given to early stopping rules and efficacy, to avoid 
Table 4 Basic requirements for establishing and running IDMCs ${ }^{139131527-29}$

\begin{tabular}{|c|c|}
\hline Basic requirements & Further details \\
\hline A clear IDMC charter & $\begin{array}{l}\text { The IDMC charter is a written document that describes the standard operating procedure of the } \\
\text { committee. } \\
\text { The IDMC charter should clearly outline the purpose of the IDMC, its role and responsibilities, the } \\
\text { operating rules, the schedule and format of meetings and the members of the IDMC and their } \\
\text { qualifications. } \\
\text { The charter defines who may attend part or all of the meetings, who the IDMC will report to and } \\
\text { its reporting process, it will describe the research data that will be monitored and how data will be } \\
\text { provided, and outline how conflict of interest will be assessed. }{ }^{39} 1327-29 \\
\text { The charter is typically drafted by the sponsor and presented to the IDMC for input and agreement. } \\
\text { The charter should be agreed on and approved by both the trial sponsor and the IDMC before the trial } \\
\text { data begins to accrue. } \\
\text { The format in which interim results are reported can be specified in the charter and is usually agreed on } \\
\text { between IDMC members and sponsors. }\end{array}$ \\
\hline $\begin{array}{l}\text { Knowledge and understanding } \\
\text { about the protocol, patient } \\
\text { population, treatment landscape } \\
\text { and monitoring plans }\end{array}$ & $\begin{array}{l}\text { All members on the IDMC should have full access to and be familiar with the oncology clinical trial } \\
\text { protocol and the data and safety monitoring plans, review study reports at the predetermined times, } \\
\text { evaluate accumulative safety and efficacy data and other trial outcomes reported in a standardised } \\
\text { format, and make recommendations on whether the trial should continue, be modified or stopped. }{ }^{315}\end{array}$ \\
\hline IDMC meeting minutes & $\begin{array}{l}\text { Minutes of each IDMC meeting should be maintained. }{ }^{327} \\
\text { The minutes may be divided in two parts - the confidential data which includes the unblinded } \\
\text { comparative data, and the summary of the discussions leading to the recommendations made by the } \\
\text { IDMC to the steering committee or the sponsor (as discussed above). } \\
\text { Generally, these minutes are kept confidential by the IDMC during the course of the trial and not } \\
\text { circulated until the completion of the trial, depending on the charter specification or institute policies. }{ }^{315}\end{array}$ \\
\hline
\end{tabular}

IDMC, independent data monitoring committee.

making the mistake of stopping a trial too soon if there is, for example, a possibility of a delayed divergence of survival curves (eg, as may be seen with immunotherapy or in case chosen surrogate endpoints in the trial do not adequately predict or reflect more definitive outcomes such as OS). ${ }^{13}{ }^{30}$ Other study results that might become available while the trial is ongoing, competing risks or changes in the standard of care during study conduct, also need to be considered for their potential impact on the ongoing clinical trial, the ethical implications and for maintaining the patients' best interests. ${ }^{15}$ This highlight the importance of selecting IDMC members with a high level of expertise and comprehensive insight in the everchanging landscape of cancer treatment.

IDMC meetings may consist of open non-confidential and closed confidential sessions (figure 2). ${ }^{13} 1015$

Prior to an IDMC meeting, a written report of the trial status, toxicity data and any appropriate outcome data should be sent by the statistician or independent Statistical Analysis Centre to the closed session IDMC members ahead of the meeting, to allow these members enough time to review the report. ${ }^{15}$ Ideally, for trial integrity purposes, it is best when interim analyses are performed by an independent statistician who is external to the sponsor and has no input on potential changes to the trial design, and serves as a 'firewall' between sponsor and IDMC (figure 3). ${ }^{324}$ This is particularly important for trials assessing evidence of effectiveness of a treatment in a blinded fashion. ${ }^{3}$ If an independent statistician is used for interim analyses and for reporting to the IDMC, the statistician does need to be very familiar with the trial design, setting and objectives. ${ }^{3}$ The advantage of using the primary trial statistician would be the familiarity with the trial, but the disadvantage would be the potential for bias or inadvertent communication of data knowledge. ${ }^{3}$ If the primary trial statistician is used for interim analyses and reporting to the IDMC, the FDA recommends that the sponsor establishes procedures for the primary trial statistician to have no further responsibility on the management of the trial (on commencement of interim analysis) and to designate another statistician to advise on the management of the trial. ${ }^{3}$

Different oncology organisations or groups may include specific specifications regarding interim analyses in their guidelines for IDMCs/DMCs. ${ }^{9} 1031$ For example, the EORTC's guideline for IDMCs specifies the minimum 


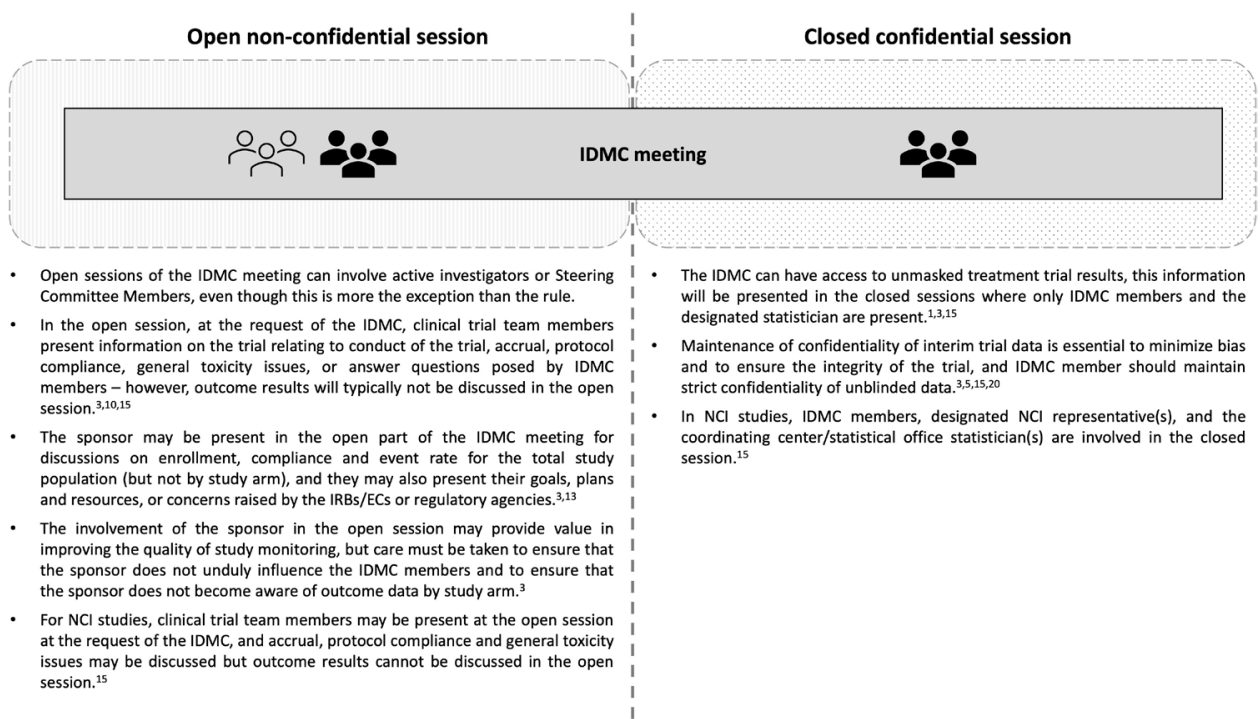

Figure 2 Open and closed sessions of the IDMC meeting. EC, ethics committees; IDMC, independent data monitoring committee; IRB, institutional review boards; $\mathrm{NCl}$, National Cancer Institute.

number of IDMC members that are required for interim reviewes of efficacy or safety (table 5). ${ }^{10}$ The IDMC/ DMC guidelines for both the EORTC and the Southwest Oncology Group (SWOG), outline the type of recommendations based on the interim analyses (table 5). ${ }^{91031}$ Interestingly, interim reviews of safety data are an important part of the EORTC's IDMC function (and a larger minimum number of IDMC members are required for interim reviews of safety compared with interim reviews of efficacy), while the SWOG's DMC reviews interim toxicity data - the study committee is primarily the responsible for this (table 5). ${ }^{10} 31$

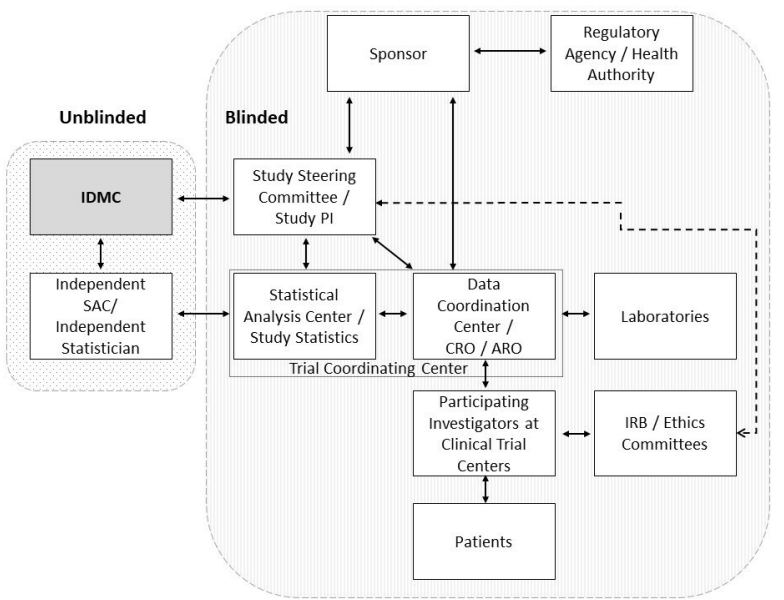

Figure 3 IDMC organisational and communication pathways flow chart. Adpated from: Flemming et $a l^{5}$ and Sirbu et $\mathrm{al}^{7}$; and Antonijevic et al. ${ }^{24} \mathrm{ARO}$, academic research organisation, $\mathrm{CRO}$, contract research organisation; IDMC, independent data monitoring committee; IRB, institutional review board; PI, principal investigator; SAC, statistical analysis centre; Sponsor, pharmaceutical or biotech company or institute or other funding agency.
A key difference between a DMC and an IDMC is that in many DMC meetings, the sponsor attends all meetings - which is not the case with an IDMC.

IDMCs primarily make recommendations to the sponsor of a given trial. Recommendations typically include items such as: whether the trial should continue unchanged, or if a modification to the protocol/treatment dosage/ patient accrual/eligibility criteria/informed consent content, etc may be needed, or if the trial needs to be terminated. ${ }^{31527}$ An IDMC meeting report with the major outcome of the meeting is provided by the chair to the trial steering committee or trial sponsor (or designee, as determined in the charter) (table 4$).{ }^{1527}$

\section{IDMC feedback and communication}

The process for communicating the recommendations from the IDMC meeting should be clearly specified in the charter (figure 3) ${ }^{20}$ Outside of what is specified in the charter, IDMC member should maintain strict confidentiality of unblinded data. ${ }^{20}$

In a typical clinical trial, the sponsor will provide feedback to the active investigators on the recommendations received from the IDMC. This feedback is usually short and concise and may simply state "continue trial as planned' or something similar.

Under certain circumstances (ie, when adverse events of concern have been observed with other trials with the same drug/class of drug/or device) regulatory authorities may request information on interim analyses from the sponsor, and with the sponsor's agreement - the IDMC chair may provide the information to the regulatory authorities. ${ }^{20}$

While there may be several institutional review boards (IRBs) / ethics committees for a clinical trial, particularly in large multicentre studies, there is only one IDMC for the trial. ${ }^{1}$ The IRBs are responsible for the safety of their patients but are unable to track all adverse events in multicentre studies, while the IDMC has access to all trial safety 
Table 5 IDMC system of decision making for interim analysis in oncology clinical trials (example for EORTC and SWOG) ${ }^{9031}$

\begin{tabular}{|c|c|}
\hline & EORTC $^{910}$ \\
\hline $\begin{array}{l}\text { IDMC review is required } \\
\text { for interim analysis }\end{array}$ & $\begin{array}{l}\text { Yes, IDMC review is mandatory for all EORTC phase II } \\
\text { and phase III trials with formal interim analyses and early } \\
\text { stopping rules that are planned and described in the } \\
\text { protocol. }\end{array}$ \\
\hline $\begin{array}{l}\text { Minimum number of } \\
\text { IDMC members required } \\
\text { for interim analysis }\end{array}$ & $\begin{array}{l}\text { For interim reviews of efficacy }{ }^{10} \text { : } \\
- \text { A minimum of three study-specific external }{ }^{10} \text { members } \\
\text { are appointed as part of the EORTC IDMC. }{ }^{10} \\
\text { For interim reviews of safety }{ }^{10} \text { : } \\
\text { - The EORTC IDMC chair + at least one permanent } \\
\text { IDMC member. }{ }^{10} \\
\text { - A minimum of two study-specific external members } \\
\text { are appointed as part of the EORTC IDMC. }{ }^{10}\end{array}$ \\
\hline
\end{tabular}

\begin{tabular}{|c|c|}
\hline & $\begin{array}{l}\text { For EORTC IDMC: } \\
\text { Stopping rules are predefined in the protocol. }{ }^{10} \\
\text { Possible protocol modifications are defined in advance. }{ }^{9} \\
\text { To avoid the inflation of a type } 1 \text { error, appropriate } \\
\text { statistical procedures are applied. }{ }^{9} \\
\text { Recommendations by the EORTC IDMC for stopping a } \\
\text { study, based on the interim analysis, may be based on }{ }^{10} \text { : } \\
\text { - Ethical considerations: that is, if it is ethical to continue } \\
\text { randomising patients to the study if there is compelling } \\
\text { evidence that the risk/benefit ratio is in favour of one } \\
\text { of the study arms over the other. }{ }^{10} \\
\text { - Futility: that is, } \\
\text { - If a conclusion will never be reached against the } \\
\quad \text { null hypothesis. }{ }^{10} \\
\text { - If the sample size might not be achieved due } \\
\text { to slow enrolment, or if sample size calculation } \\
\text { assumptions are not achievable. }{ }^{9} \\
\text { - Safety issues that might require discontinuation of the } \\
\text { treatment regimens in the study. }{ }^{10} \\
\text { - Other parameters that may have been predefined as } \\
\text { stopping rules in the study protocol. }{ }^{10} \\
\text { At the interim analysis, the EORTC IDMC may also make } \\
\text { recommendations }{ }^{10} \text {. } \\
\text { - Whether modifications to the treatment regimen or } \\
\text { study protocol are required (which may be made for } \\
\text { safety or patient enrolment reasons). }{ }^{10} \\
\text { - Whether a modification is needed in the sample size. }{ }^{10} \\
\text { - Whether a phase II study should continue (or be } \\
\text { expanded) into a phase III study. }{ }^{10}\end{array}$ \\
\hline
\end{tabular}

System of decision
SWOG $^{31}$

- SWOG requires a DSMC (ie, DMC) to monitor all SWOG-coordinated phase III and randomised phase II oncology trials, ${ }^{31}$ thus, if such trials have planned interim analyses, a DMC will be monitoring the study.

- Not specified, however the voting quorums for a SWOG DSMC/DMC require that the majority do not belong to SWOG. ${ }^{31}$

\section{For SWOG DSMC/DMC:}

- Decision rules are predefined in the study protocol. $^{31}$

- Prior to their implementation, the SWOG DSMC reviews major study modifications that are proposed by the study committee, which include ${ }^{31}$ :

- Stopping of the study/study termination. ${ }^{31}$

- Discontinuing a study arm based on toxicity results or other reported trials. ${ }^{31}$

- Increasing the sample size. ${ }^{31}$

- Recommendations by the SWOG DSMC may be made, based on the interim analyses, on ${ }^{31}$ :

- Whether the trial should be stopped for either patient safety reasons, inferior therapy, or closed early due to slow accrual. ${ }^{31}$

- Whether the trial should be changed (which could be for patient safety reasons, or extension of accrual, etc). ${ }^{31}$

- The SWOG DSMC makes their recommendations based on the study results and also on available data from other related studies. ${ }^{31}$

- For studies that the network groups or other organisations perform, the SWOG DSMC also determine if group studies need to be stopped or changed (based on reviewing the reports of the study and study committee recommendations). ${ }^{31}$

- The SWOG DSMC reviews interim toxicity data, however, the study committee is primarily responsible for this. ${ }^{31}$

- Recommendations by the SWOG DSMC are provided to the SWOG group chair. ${ }^{31}$
Communication

- Recommendations by the EORTC IDMC are provided to the study management group and study steering committee. $^{10}$

DMC, data monitoring committee; DSMC, data and safety monitoring committee; EORTC, European Organisation for Research and Treatment of Cancer; IDMC, independent data monitoring committee; SWOG, Southwest Oncology Group.

data. ${ }^{1}$ Typically the IRBs will receive reports in connection with IDMC actions through the trial sponsor or SSC. ${ }^{1}$ However, in some instances (eg, for certain institutional studies) the institutional IDMC might provide the report directly to the IRB or via the investigator to the IRB but generally, the IRB does not receive the IDMC reports direct from an IDMC external to its institute. ${ }^{4}$

\section{Potential limitations to the functioning of IDMCs in oncology trials}

In the setting of clinical trials involving rare malignancies, it can be exceedingly difficult to identify suitable members for IDMCs as too few people may have the required expertise and level of independence.

There are also a limited number of people who are experienced, or trained, in participating in IDMCs for oncology clinical trials. At the same time, there is an increasing demand for IDMCs. There is a tremendous need to provide either formal training, or mentoring, to inexperienced IDMC members. Understanding of the responsibilities and functioning of the IDMC, as well as the statistics and stopping rules involved in the trial is crucial for the success of the IDMC. 
While the IDMC members can be in the unique position of having access to unblinded data, they have the obligation of maintaining the integrity of the study when communicating with the sponsor. If the feedback from the IDMC meeting to the sponsor requires more than a simple statement and some details are included, or in an open session, it could become an issue in keeping the interim data of a study blinded particularly in trials with a 2:1 randomisation (where it can easily be seen, based on recruitment figures, which arm reflects the investigational treatment). Careful thought is needed in these communications to the sponsor to ensure that the data remain blinded.

Many oncology studies require a very long time period to accurately assess survival and mortality rates. This may pose a problem for continuity in maintaining the same membership of the study's IDMC over several years. To address this problem, the charter could include criteria for (periodic) revision of the IDMC's membership.

\section{RECOMMENDATIONS}

IDMCs should be used for all randomised oncology clinical trials, but other trials in oncology can benefit as well from independent study oversight.

There are no regulatory requirements for IDMC training, but because of the importance of the role of the IDMC and the complexities of the decisions that may be required of the IDMC members, it is recommended that IDMC members either have previous IDMC experience or have some training/mentorship/or guidelines provided to them. ${ }^{45}$ It is particularly important that the committee chair has prior IDMC experience. ${ }^{20}{ }^{26}$ However, the inclusion of at least one member without prior experience on IDMCs for oncology clinical trials, particularly early career individuals, should be encouraged for growing the pool of 'qualified' IDMC member to meet the increasing demand. ${ }^{162026}$

Training of inexperienced members could include workshops, online trainings or through books/journal articles/case studies and hands-on training through partnering with an experienced IDMC mentor. ${ }^{616}{ }^{20}$ Figure 4 shows different options for IDMC training which include: (1) self-study of the pertinant regulatory guidelines and the study group or organisational guidelines for the specific clinical trial; (2) self-study of books or published journal articles or case studies on IDMCs/DMCs (google search terms for books can include: DMCs in clinical trials/DSMCs in clinical trials/data monitoring in clinical trials); (3) in-person or on-line training courses on IDMCs/DMCs (google search terms for training/courses: DMC training/DMC courses/DMC programmes/IDMC training programmes); (4) mentorship or 'on-the-job' training where an experienced IDMC member trains an inexperienced IDMC member from the start (including requirements, responsibilities, guidelines) and through the meeting and decision-making processes for a specific clinical trial. Development should include both IDMC training and information on oncology clinical trial methodology and complexities, key statistical concepts and stopping rules, and the use of IDMC/DMC case studies could be useful in showing what works well in the decision making process and errors to avoid (figure 5) ${ }^{1620} \mathrm{It}$ would be desirable to establish formal criteria for potential IDMC members and chairs, based on well documented training routines, but this is not the case as of yet. A recommendation for formal IDMC training is the development of a certificate or accredited programme with a central registry (including disease or tumour-specific specialty) to allow easy access to a pool of IDMC/DMC 'qualified' members.

Other training could include biomedical ethics for IDMC members, and meeting leadership and consensus development training for the chair. ${ }^{516}$

An IDMC could also be set up to be used to cover a portfolio of related clinical trials instead of a single trial only.

IDMC members need to be adequately prepared to take on their roles and responsibilities, and to adhere to important standards as outlined in the IDMC charter. ${ }^{23}$ There is a need for further training of the independent statistician or Statistical Analysis Centre, sponsors and IDMC members on what to expect from the statistical data analysis, and how it will be carried out to maintain confidentiality. ${ }^{17} 32$ Early stopping rules should be clearly explained, understood and agreed to by all IDMC members. ${ }^{17}$

At the start of each clinical trial, the IDMC members and the independent statistician or the Statistical Analysis Centre should also have a clear understanding and awareness of the study protocol and IDMC Charter for that particular study. ${ }^{16}$ They should have a deep understanding of the highly confidential character of most

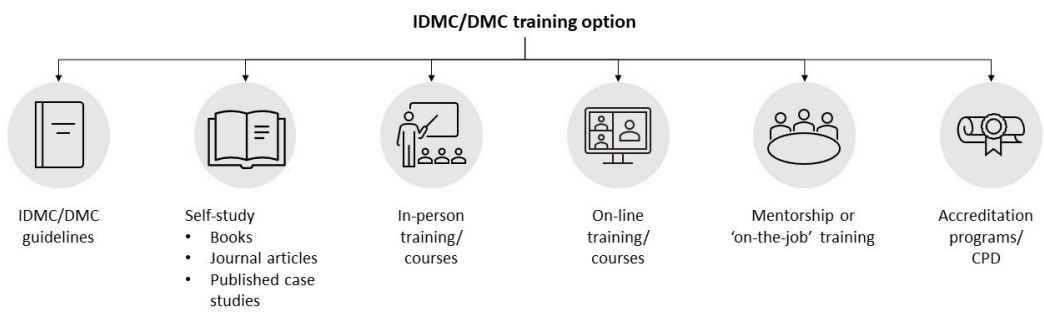

Figure 4 Different IDMC/DMC training options for developing experienced IDMC members. CPD, continuing professional development; DMC, data monitoring committee; IDMC, independent data monitoring committee. 


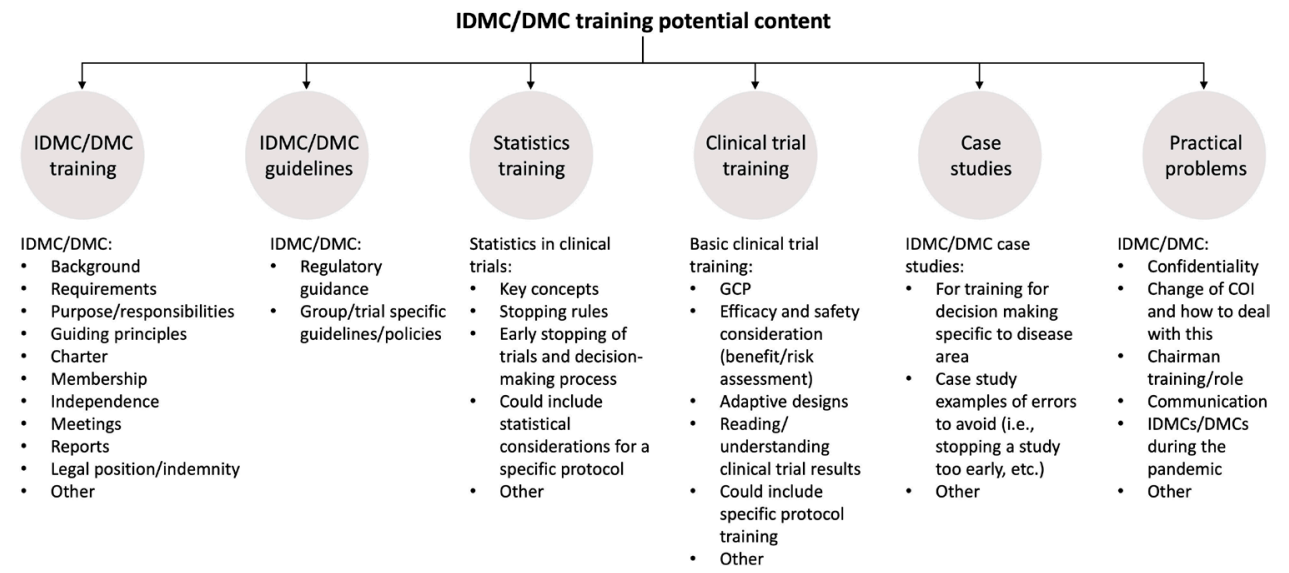

Figure 5 Potential types of content for incorporation into IDMC/DMC training. COI, conflict of interest; DMC, data monitoring committee; GCP, Good Clinical Practice; IDMC, independent data monitoring committee.

IDMC functions and the potential impact of conflicts of interest.

\section{CONCLUSION}

While IDMCs for oncology clinical trials have many similarities with other therapeutic area IDMCs, such committees in oncology are of particular importance because of the size of cancer trials, the long duration of many of these trials with survival outcome assessments, the potential toxicity of the treatment, the severity of the disease, the high level of innovation in many cancer trials and associated uncertainties with these novel approaches, and many trials include preplanned interim analyses. Oncology clinical trials with survival or disease progression as endpoints should use IDMCs. Patient safety and trial integrity remain key focal points of IDMCs. IDMC members should be selected based on their expertise and their ability to fulfil their role and responsibilities on the IDMC. The increased use of IDMCs over the past decades highlights the lack of availability of qualified or experienced IDMC members and the need for developing future generations of IDMC members, through training and mentorship. IDMC members need to work to a clear Charter, be free of significant conflicts of interests, and have a good understanding of the trial protocol and stopping rules which may also need to incorporate considerations for a potential delay in the divergence of survival advantages in the oncology trial.

\section{Twitter P Schöffski @schoffski}

Acknowledgements PS funded editorial/medical writing support, and J. 0'Regan (Bingham Mayne and Smith) provided medical writing/editing support and coordinated the final submission.

Contributors PS proposed and developed the manuscript. PS approved the final version of this manuscript and was responsible for the decision to submit the manuscript.

Funding The author has not declared a specific grant for this research from any funding agency in the public, commercial or not-for-profit sectors.

Competing interests PS has been involved in numerous DMCs/IDMCs for various oncology clinical trials with both commercial and academic sponsors. He has participated as both a member and chair on these DMCs/IDMCs.
Patient consent for publication Not required.

Provenance and peer review Not commissioned; externally peer reviewed.

Open access This is an open access article distributed in accordance with the Creative Commons Attribution Non Commercial (CC BY-NC 4.0) license, which permits others to distribute, remix, adapt, build upon this work non-commercially, and license their derivative works on different terms, provided the original work is properly cited, appropriate credit is given, any changes made indicated, and the use is non-commercial. See: http://creativecommons.org/licenses/by-nc/4.0/.

ORCID iD

P Schöffski http://orcid.org/0000-0001-5980-030X

\section{REFERENCES}

1 Sartor O, Halabi S. Independent data monitoring committees: an update and overview. Urol Oncol 2015;33:143-8.

2 National Institutes of Health $(\mathrm{NIH})$ guide. volume 8 number 8, 1979. Available: file:///C:/Users/Admin/Documents/My\%20documents/ From\%20PC\%203\%20Sept\%202018/2020\%20-\%202019\%20 Schoffski\%20-\%20data\%20monitoring\%20article/From\%20 internet\%20-\%20marked\%20up/NIH\%20guide.\%20Volume\%208\% 20number\%208,\%20June\%205,\%201979.pdf [Accessed 27 Feb 2020].

3 U.S. Department of Health and Human Services. Food and Drug Administration. Guidance for clinical trial sponsors. Establishment and operation of clinical trial data monitoring committees. March 2006. omb control No. 0910-0581 (expiration date: 10/31/2021). Available: https://www.fda.gov/media/75398/download [Accessed 27 Feb 2020].

4 CTSA Collaborative DSMB Workgroup. DSMB training manual. Medford, MA: Tufts Digital Library, 2018. https://www.tuftsctsi.org/ research-services/regulatory/data-and-safety-monitoring-boardtraining-manual-for-investigator-initiated-studies/

5 Fleming TR, DeMets DL, Roe MT, et al. Data monitoring committees: promoting best practices to address emerging challenges. Clin Trials 2017;14:115-23.

6 Hess CN, Roe MT, Gibson CM, et al. Independent data monitoring committees: preparing a path for the future. Am Heart $J$ 2014:168:135-41.

7 Sirbu C, Lucas BD, Broce M, et al. Data \& Safety Monitoring Boards in Industry-Sponsored Clinical Trials. J Clin Res Best Pract 2008;4 http://citeseerx.ist.psu.edu/viewdoc/download?doi=10.1.1.536. 7276\&rep=rep $1 \&$ type $=$ pd

8 World Health Organization (WHO). UNICEF / UNDP / WORLD BANK / WHO special programme for research and training in tropical diseases (TDR). Operational guidelines for the establishment and functioning of data and safety monitoring boards. 2005, TDR/GEN/ Guidelines/05.1. Available: https://www.who.int/tdr/publications/tdrresearch-publications/operational-guidelines/en/ [Accessed 03 Mar 2020].

9 European Medicines Agency (EMEA). Guideline on data monitoring committees. Doc. Ref. EMEA/CHMP/EWP/5872/03 Corr, 2005. Available: https://www.ema.europa.eu/en/documents/scientific- 
guideline/guideline-data-monitoring-committees_en.pdf [Accessed 03 Mar 2020].

10 Collette L. European Organisation for Research and Treatment of Cancer (EORTC). Independent data monitoring committees for EORTC studies. POL004 version 3.0, 2016. Available: https://www.eortc.org/app/uploads/ 2017/03/POL004-v-3-0.pdf [Accessed 09 Mar 2020].

11 Sydes MR, Spiegelhalter DJ, Altman DG, et al. Systematic qualitative review of the literature on data monitoring committees for randomized controlled trials. Clin Trials 2004;1:60-79.

12 National Institutes of Health $(\mathrm{NIH})$. Further guidance on a data and safety monitoring for phase I and phase II trials. Notice: OD-00-038, 2000. Available: https://nccih.nih.gov/node/9221 [Accessed 10 Mar 2020].

13 Wittes J, Schactman M. On independent data monitoring committees in oncology clinical trials. Chin Clin Oncol 2014:3:40-50.

$14 \mathrm{ICH}$ Harmonised Tripartite Guideline: guideline for good clinical practice E6 (R1). International Conference on Harmonisation of technical requirements for registration of pharmaceuticals for human use, 1996. Available: https://www.gmp-compliance.org/guidelines/ gmp-guideline/ich-e6r2integrated-addendum-to-ich-e6r1-guidelinefor-goodclinical-practice [Accessed 20 Apr 2020].

15 National Cancer Institute (NCl). Policy of the National Cancer Institute for data and safety monitoring of clinical trials. Approved by the $\mathrm{NCl}$ Clinical and Translational Research Operations Committee (CTROC), 2014. Available: https://deainfo.nci.nih.gov/grantspolicies/datasafety. pdf [Accessed 10 Mar 2020].

16 Calis KA, Archdeacon P, Bain RP, et al. Understanding the functions and operations of data monitoring committees: survey and focus group findings. Clin Trials 2017;14:59-66.

17 Ellenberg SS. Protecting clinical trial participants and protecting data integrity: are we meeting the challenges? PLOS Med 2012;9:e1001234.

18 Lin M, Chow S-C. Data monitoring committees in adaptive clinical trials. Clin Investig 2013;3:605-7.

19 DeMets DL, Ellenberg SS. Data monitoring committees - expect the unexpected. N Engl J Med 2016;375:1365-71.

20 Filippatos GS, de Graeff P, Bax JJ, et al. Independent academic data monitoring committees for clinical trials in cardiovascular and cardiometabolic diseases. Eur J Heart Fail 2017;19:449-56.

21 Cuzick J, Howell A, Forbes J. Early stopping of clinical trials. Breast Cancer Res 2005;7:181-3.
22 Collette L. European Organisation for Research and Treatment of Cancer (EORTC). Permanent Independent Data Monitoring Committee (IDMC) for EORTC studies. IDMC charter. Version 1.0, 2015. Available: https://www.eortc.org/app/uploads/2017/06/IDMCcharter.pdf [Accessed 12 Mar 2020].

23 Snowdon C, Brocklehurst P, Tasker RC, et al. "You have to keep your nerve on a DMC." Challenges for data monitoring committees in neonatal intensive care trials: Qualitative accounts from the BRACELET Study. PLoS One 2018;13:e0201037.

24 Antonijevic Z, Gallo P, Chuang-Stein C, et al. Views on emerging issues pertaining to data monitoring committees for adaptive trials. Ther Innov Regul Sci 2013;47:495-502.

25 Radiation Therapy Oncology Group (RTOG). Phase III data monitoring committee, 2005. Available: https://www.rtog.org/ LinkClick.aspx?fileticket=R3fG2hYgdqE=\&tabid=183 [Accessed 16 May 2020]

26 Australian Government National Health and Medical Research Council. Data safety monitoring boards (DSMBs), 2018. Available: https://www.australianclinicaltrials.gov.au/sites/default/files/content/ For\%20researchers/Data\%20Safety\%20Monitoring\%20Boards_1. pdf [Accessed 16 May 2020].

27 Harvard Catalyst, The Clinical and Translation Science Center. Data and safety monitoring guidance, 2018. Available: https://catalyst. harvard.edu/pdf/regulatory/DSMB-P_Guidance.pdf [Accessed 26 May 2020].

28 Dixon DO, Freedman RS, et al, SCT Working Group on Data Monitoring. Guidelines for data and safety monitoring for clinical trials not requiring traditional data monitoring committees. Clin Trials 2006;3:314-9.

29 DAMOCLES Study Group, NHS Health Technology Assessment Programme. A proposed charter for clinical trial data monitoring committees: helping them to do their job well. Lancet 2005;365:711-22.

30 Hoos A. Evolution of end points for cancer immunotherapy trials. Ann Oncol 2012;23 Suppl 8:viii47-52.

31 Southwest Oncology Group (SWOG). Policy memorandum no 21, data and safety monitoring policy, 2013. Available: https://www. swog.org/sites/default/files/docs/2017-10/Policy21.pdf [Accessed 30 Aug 2021].

32 DeMets $\mathrm{D}$. The independent statistician model: how well is it working? Clin Trials 2018;15:329-34. 